\title{
Meta-Learning Applied to the Selection of the Classification Methods in Industrial Images
}

\author{
Luis Fernando Marin Sepulveda* Aristófanes Correâ Silva* \\ João Otávio Bandeira Diniz* \\ * Department of Electrical Engineering, Federal University of \\ Maranhão - UFMA, MA, (e-mail: lfmarins@unal.edu.co, \\ ari@dee.ufma.br, joao.bandeira@nca.ufma.br)
}

\begin{abstract}
In industrial production, control of quality of product and analysis of state of the staff in charge are important factors, nevertheless, the collection and analysis of data imply large amounts of time and may involve a risk to health of the staff in case of quality control, to deal with these tasks, image capturing and classification tools have been used, however, there is a challenge to identify the most appropriate classification method when taking into account the type of image being studied, the challenge is greater when it is necessary for a system to process different products with different classification objectives. This paper presents a methodology based on Meta-Learning and CNN for the identification of the appropriate methods of classification of industrial images. As an object of study images of hot-rolled steel strip, shear pad of wagon train, welds x-rays, aluminum wheel x-rays and human faces were used, obtaining $96 \%$ accuracy, $99.7 \%$ AUC and $96.5 \%$ F- measure.
\end{abstract}

Keywords: Meta-Learning, Image Processing, Machine Learning, Image Classification, CNN.

\section{INTRODUCTION}

Currently large amounts of data are obtained, in part due to advances in the capture, transmission, storage and processing devices, however, the challenge is to analyze all this new amount of data to build descriptors, classifiers, diagrams and other forms of valuable models (Brazdil, 2018),(Weiss, 2016), nevertheless, there is no method that can solve all the needs, instead there is a large number of methods to solve specific tasks, some based on Machine Learning; this great set of available methods produces the need for method recommendation strategies for a specific task based on its nature and the features of the data (Smith, 2014). Considering a scenario in which there are multiple methods that can perform a task, the challenge is to identify which is the most appropriate; without a recommendation strategy, one option is to test each method independently and compare its performance, that presents the disadvantage that for each new type of dataset under study it is necessary to repeat the tests, even when the task is the same, this happens because each dataset has inherent features that are used by methods to provide results (Pimentel, 2019). A recommendation strategy has the advantage of avoiding reprocessing, for its construction, it is possible to use features of dataset and result of different methods to establish a performance relationship, where the relationship is known as Metaknowledge and allows to predict the performance of new datasets under study (Cunha, 2018),(Rahman, 2017).

Meta-Learning uses Meta-knowledge as data or experience to identify the method that presents a better performance for a specific task, it can be used in the field of image processing with different objectives, as is the case of segmentation, classification and identification (Cheplygina, 2017),(Amiri, 2014). In the industry there are processes that are being automated using image processing and require identifying the most appropriate method for each task, for example, to detect product defects in a production line or in a welding, it is necessary to determine which classification method is more efficient (Mery, 2015).

The aim of this work is to demonstrate the efficiency of the use of Meta-Learning for identification of most appropriate classification methods for different types of industrial images, understanding as more appropriate the method that presents a better performance for a type of image with respect to the evaluation measures. The main contributions of this work are: First, a new application of MetaLearning in industrial images for selection of classification methods. Second, the application of the Convolutional Neural Network (CNN) as a method for classifying and characterizing the Meta-Model for hot-rolled steel strip, the shear pad of wagon train, welds x-rays, aluminum wheel x-rays and human faces images.

The paper is organized as follows: Section 2 presents an analysis of the state of the art of Meta-learning. Section 3 introduces the datasets used in this work. Section 4, exposes the proposed methodology. Section 5 presents results and discussions. Finally, section 6, concludes the document.

\section{RELATED WORK}

In this section will be presented works related to the proposed method, and important concepts about meta- 
learning will be presented. These works were fundamental for the construction of the proposed methodology.

Meta-Learning was used in Pimentel (2019) to predict the performance of different clustering algorithms applied to a dataset, using as a learning source a set of Meta-Features that describe the dataset. This paper proposes a new set of Meta-Features that combines measures of correlation and dissimilarity, and the experiments used 219 datasets collected from Open Machine Learning (OpenML). The results show that the use of the k-Nearest Neighbor (k$\mathrm{NN}$ ) and Random Forest (RF) techniques for the MetaModel presents a better clustering quality when using the proposed method. This paper presents an application of Meta-Learning for the selection of clustering algorithms, proposing a new set of Meta-Features, however, the methods under study are limited to a single objective task.

The methodology presented in our proposed work differs in the objective of the methods under study, the form of characterization of the dataset and in the type of data analyzed, it also has the advantage of allowing the methods under study to have different objective tasks.

In Mantovani (2015) Meta-Learning is used with the objective of identifying the appropriate configuration of the Hyper-parameters of the Support Vector Machines (SVM) technique, based on the features of the dataset. For the Meta-Model, six classification techniques were tested: kNN, Nave Bayes (NB), RF, J48 Decision Tree (J48), SVM and Multilayer Perceptron (MLP), in addition 21 datasets were used for training and 145 datasets for test, using 10 -fold cross-validation. When comparing the results with the configurations usually used, the new set of Hyperparameters produces significant improvements. This paper presents an application of Meta-Learning for the identification of suitable configuration parameters of the SVM technique according to the features of the datasets. Given the objective of analysis, the article is limited to a single classification technique as an object under study.

The methodology presented in our proposed work differs in the objective of the methods under study, the form of characterization of the dataset and in the type of data analyzed, it also has the advantage of using multiple classification techniques with different configurations as object under study.

Meta-Learning is used in Campos (2017) for the selection of image segmentation algorithm. For the Meta-Model were examined the techniques: NB, linear classifier, C4.5 Decision Tree, k-NN, RF, Neural Networks using model averaging and SVM. The segmentation methods under study are: k-NN, Otsu's thresholding and SVM. 44 features were used based on color, histogram, frequency domain, texture, image quality and contrast, applied to four datasets with different segmentation objectives. The Random Forest technique presents the best performance, obtaining average of $88.7 \%$, F-Measure of $81.4 \%$, Area Under the ROC curve (AUC) of 93.7\%, true negative rate of $88.5 \%$ and true positive rate of $81.3 \%$. This paper presents a Meta-learning application for the selection of the most suitable segmentation algorithm based on the extracted features.
The methodology presented in our proposed work has a similar logic of development, however, differs in the objective of the methods under study, the form of characterization of the dataset and in the type of image analysed, in addition the results obtained in this proposed work exceed to those reported.

\section{DATASETS}

Meta-Learning is used in this work for the selection of the most appropriate classification methods for five datasets with different types of industrial images, each dataset has a specific objective: for hot-rolled steel strip the aim is the classification of defects; for the shear pad of wagon train the objective is the classification of the state of the pad; for welds x-rays the target is the identification of geometric faults; for aluminum wheel x-rays the objective is the detection of defects; finally for human faces the objective is the classification of emotion based on expression.

The NEU surface defect dataset was created by Northeastern University, contains six types of surface defects of Hot-rolled Steel Strip: rolled-in scale, patches, crazing, pitted surface, inclusion and scratches. The dataset consists of 1800 grayscale images with 300 samples of each type of defect, each image has a resolution of 200x200 pixels. The NEU Surface defect dataset includes different difficult challenges that include image defects given the influence of lighting and the change in materials (Song, 2013).

The Shear pad of wagon train dataset based on Rocha (2017), is constituted by 1081 colorful images, with a resolution of $640 \times 480$ pixels and include the whole wagon truck, each image contains the elements: Pad, suspension springs and roller bearing screw set.

The Welds X-ray dataset was taken by the BAM Federal Institute for Materials Research and Testing, Berlin, Germany and is part of X-ray images for X-ray testing and Computer Vision (GDXray). The dataset contains 88 images arranged in 3 series, and have 641 defects. the images are in 8-bit gray scale with a pixel size of 40.3 microns (630 dpi) (Mery, 2015).

The Aluminum wheel X-ray dataset consists of 72 images taken with 5-degree rotation and using an image intensifier, additionally includes defect annotations and calibration. The selected dataset is a subset of the Castings group that contains 2727 images and belongs to GDXray (Mery, 2015).

The Human Face dataset is made up of 300 images of human faces, selected from the MIRFLICKR-25000 collection. The dataset is made up of images with different size, lighting and sharpness characteristics, and includes colourful and gray scale images. The faces in the images belong to different people that vary in gender and age (Huiskes, 2008),(Huiskes, 2010).

\section{PROPOSED METHODOLOGY}

The methodology of this work consists of three steps: First, Classification Method Selection, based on the literature is identified the most appropriate classification method for each type of dataset; the result of this step together 
with the Industrial Image Dataset, make up the Metaknowledge that is contained in the Meta-Data. Second, Classifier Evaluation for Meta-Model, this step uses different Convolutional Neural Network (CNN) configurations from Classifier CNN Methods to process the MetaData and identify the configuration that presents a better performance; the result of this step together with the Meta-Data make up the Meta-Model. Finally, Meta-Model Validation, the proposed methodology is validated using a new test set. The proposed methodology can be seen in Figure 1.

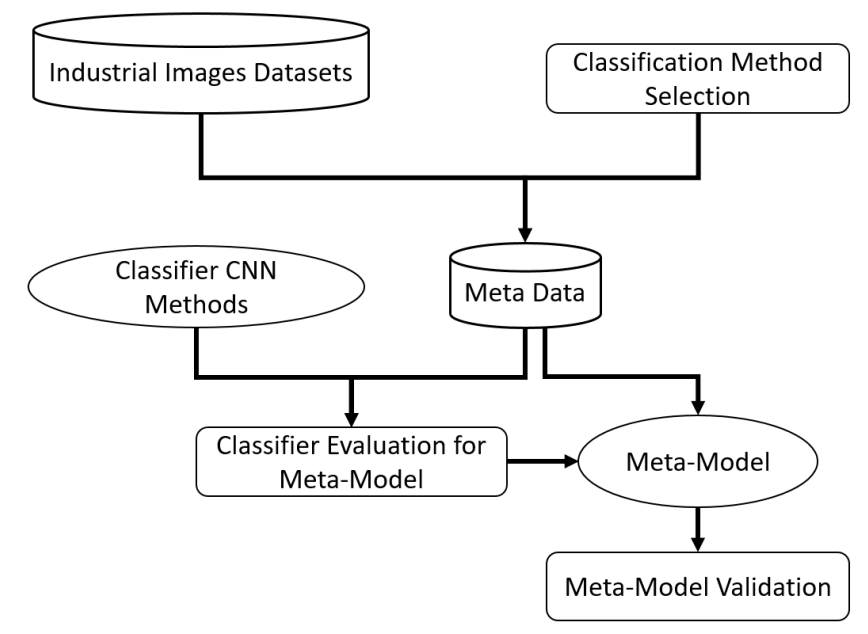

Figure 1. Proposed Methodology.

\subsection{Classification Method Selection}

At this step a classification method based on the literature is selected for each dataset, the selected methods do not have to be similar, this quality implies that each method can use a different characterization form to represent the dataset. The information of each selected classification method, as well as the type of characterization used, is considered experience and is part of the Meta-data. It is necessary to clarify that in this step the classification methods found are not implemented or the recommended characterization techniques are applied, both are only identified and established for each dataset.

For NEU surface defect dataset according to Song (2013), the SVM technique is identified as the most suitable for performing defect classification, together with the use of a proposed new characterization method called adjacent evaluation completed local binary patterns (AECLBP).

SVM is a supervised machine learning algorithm that is used for classification and regression tasks, the algorithm consists in the creation of a Hyperplane with an equidistant separation between the closest samples of each class (Vapnik, 1995). AECLBP decomposes the local differences of the image into two complementary components: the sign $\left(S_{p}\right)$ and the magnitude $\left(m_{p}\right)(1)$, then two operators AECLBP_S (2) and AECLBP_M (3) are used to perform the coding.

$$
S_{p}=S\left(a_{p}-g_{c}\right), m_{p}=\left|a_{p}-g_{c}\right|
$$

where $g_{c}$ is the value of the gray scale of the center point, $a_{p}$ is the average value of the evaluation window $p$ th excluding the value from the center of the window.

$$
\text { AECLBP_S } S_{P, R}=\sum_{p=0}^{P-1} S\left(a_{p}-g_{c}\right) 2^{P}, S(x)=\left\{\begin{array}{l}
1, x \geq 0 \\
0, x<0
\end{array}\right.
$$

where $P$ points spaced equidistantly around a circle of radius $R$.

$$
\text { AECLBP_M } M_{P, R}=\sum_{p=0}^{P-1} t\left(m_{p}, c\right) 2^{P}, t(x, c)=\left\{\begin{array}{l}
1, x \geq c \\
0, x<c
\end{array}\right.
$$

where $c$ is the average value of $m_{p}$ of the whole image.

For Shear pad of wagon train dataset according to Rocha (2017) the use of CNN in conjunction with SVM to replace the softmax layer is efficient for pad classification, when used in grayscale images.

$\mathrm{CNN}$ is a neural network that uses convolution in at least one of its layers, CNN extracts features from the original data if these are arranged in a matrix; each neuron of the convolution layer can applies a different filter, the resulting data of the convolution layer are used as input for the next layer that performs a sub-sampling process, before or after starting the sub-sampling an activation function is performed. CNN can be constructed using different configurations of the convolution and sub-sampling layers, which vary in number, size of the matrices and number of neurons. The final layer consists of a full connected network who is responsible for carrying out the classification (Lecun, 2015). In the proposed CNN the first convolution layer has 32 neurons with $3 \times 3$ kernel, step 1 and ReLU activation. the next convolution layer has 64 neurons, with $3 \times 3$ kernel, step 3 and ReLU activation. The fully connected layer has 128 neurons with ReLU activation and $50 \%$ abandonment, the final layer consists of SVM.

For Welds X-ray dataset according to Baldassari (2017) the Hidden Markov Model (HMM) contributes to the identification of geometric faults, when using as input data the information obtained after applying the method of Gaussian mixtures on the features resulting from applying the Principal Component Analysis (PCA) method on images.

The Hidden Markov Model (HMM) is a stochastic process based on two levels of uncertainty: a random observation process associated with each hidden state and a Markov chain that characterizes the probability relationship between the states. HMM is used for the prediction of states based on the information of the current state and the conditional probability of the hidden states, and is also used for pattern recognition (Rabiner, 1989). The PCA has the purpose of transforming a set of variables, calls of originals, into a new set of variables called principal components. The new variables are linear combinations and are constructed according to the order of importance in terms of the total variability that they collect from the sample (Jolliffe, 2002). The Gaussian mixture model 
(Reynolds, 2015) is a parametric probability density function that represents the density of sub populations that have a Gaussian distribution (4).

$$
P(x \mid \theta)=\sum_{i=1}^{k} w_{i} g\left(x \mid u_{i} \sum i\right)
$$

where $x$ is a D-dimensional continuous-valued data vector, $w_{i}$ are the mixture weights and $g\left(x \mid u_{i} \sum i\right)$ are the component Gaussian densities.

For Aluminum wheel X-ray dataset the method proposed by Mery (2002) presents efficient results for the automatic detection of defects in sequential images taken with rotation of the piece in small intervals of time. The method basically consists of two steps: identification and monitoring of potential failures.

The identification of potential faults uses two features of the defects to perform their individualization: First, a defect can be considered as a connected subset of the image. Second, there is a significant difference in the intensity of the gray level between the fault and its neighbours, however, it is necessary to perform a pre-processing to identify the signal-to-noise ratio and establish a classification threshold for the intensity of the gray level. To carry out the identification of potential faults, two processes are applied: edge detection, where a Laplacian of Gaussian kernel and a zero-crossing algorithm are used to detect the edges present in the images. Segmentation and classification of potential faults, regions with closed limits whose average gray level is $2.5 \%$ greater than the average gray level of their neighbours and whose area is greater than 15 pixels are labelled as potential faults. The tracking of potential faults allows separating the real faults from the false positives, so that a failure is considered true, must comply with the follow-up in three steps: Matching in two views, where the faults detected in two images of the same piece with rotation, they coincide in relative position of the object and extracted features. Tracking in more views, where the path of the fault is established in three images of the same piece with rotation. Finally, verification, where minimum squares are used to establish the centers of gravity of the faults, projecting a point in each trajectory.

For Human Face dataset according to Rivera (2017) the use of features based on Units of Action (AU) and the classifier k-NN allows the detection of six basic facial expressions.

AU allow the description of perceptible facial muscle movements based on AU functions, in Rivera (2017) 20 action units are used: two InnerBrowRaiser, two OuterBrowRaiser, two BrowLowerer, UpperLipRaiser, two LipCornerPuller, two CheekPuffer, two LipStretcher, two LipCornerDepressor, jawLowerer , two EyesClosed, two JawLeftRight. The AU can take values between 1 and -1 , and are treated as a vector of 20 dimensions. The employed AUs are written in the facial action coding system proposed by Ekman (1976). K-nearest neighbor kNN (Deng, 2016) is used as a classification technique, in which the information of the training samples is taken and $\mathrm{k}$ samples of each class are selected, using this information and based on the separation of the samples are defined the regions that separate the classes, in Rivera (2017), a 20-dimensional system is used to represent each sample based on the AU features, the Euclidean distance between the samples is obtained using (5).

$$
D\left(p_{1}\left(x_{1}, \ldots x_{n}\right), \ldots, p_{n}\left(y_{1}, \ldots y_{n}\right)\right)=\sqrt{\sum_{i=1}^{N}\left(x_{i}-y_{i}\right)^{2}}
$$

where $D$ is the Euclidean distance, $p_{n}$ are the samples, $x_{i}$ and $y_{i}$ are AU features.

\subsection{Classifier Evaluation for Meta-model}

In this step, different CNN configurations are tested to identify the one that presents a better performance with respect to the evaluation measures. The selected configuration is used as a classification method for the Meta-Model. Each configuration is tested using the same Meta-Data, which contains a set of training images, information of the most appropriate classification method for each type of image and its characterization method, according to the information obtained in the step of Classification Method Selection (Section 4.1).

In this work, CNN is selected as a classification method for the Meta-Model because of its ability to extract features directly from the image and perform the classification task without the intervention of alternative characterization methods (Chaib, 2017). Given the variety of configurations that can be used for the classification task, identifying the most appropriate implies tests, two configurations known in the literature and fourth proposed configurations are tested and compared:

LeNet Network (Ben, 2017) is a well-known convolutional neuronal network; the first layer is convolutional and consists of 20 neurons with a $5 \times 5$ matrix and RELU activation; the second layer is subsampling with a $2 \times 2$ matrix and maximum value selection function; the third layer is convolution and is made up of 50 neurons with a 5x5 matrix and RELU activation; the fourth layer is subsampling and is similar to the second layer; the fifth layer is dense with RELU activation; the last layer is classification.

VGG16 Network (Simonyan, 2014) is a convolutional neural network architecture named as the Visual Geometry Group from Oxford; VGG16 has 13 convolutional layers, five subsampling layers and one output layer. The first two convolution layers are made up of 64 nodes each one, the next two are made up of 128 nodes each one, the next three nodes each one have 256 nodes, and finally the last six layers are made up of 512 nodes each one; all layers use $3 \times 3$ convolution kernels and RELU activation function.

The first proposed configuration is designed to be simple and determine if more than one convolution layer is necessary and if a low number of neurons is adequate to perform the characterization; the first convolution layer is made up of 20 neurons with a 5x5 matrix and RELU activation, the second subsampling layer uses a $2 \times 2$ matrix and a maximum value selection function, the last layer is full connected and is responsible for classification. 
The second proposed configuration is based on the first, changing the size of the convolution matrix in order to determine if there is a loss of relevant information; the matrix the first convolution layer is $3 \times 3$ with a RELU activation function.

The third proposed configuration is based on the first, changing the size of the subsampling matrix to determine its effect on the performance of the classification, the subsampling layer uses a $3 \times 3$ matrix and the maximum value selection function.

The fourth proposed configuration performs a combination of the two previous ones, in order to determine the effects on the performance; the first convolution layer is made up of 20 neurons with a RELU activation, and a $3 \times 3$ convolution matrix; the second subsampling layer uses $3 \times 3$ matrix and maximum value choice function; the final layer is full connected and performs the classification.

The step begins with the creation of the training set, containing the images and the class labelling information. Then, each of the six configurations of the CNN method is tested using the Meta-Data. The performance of each CNN configuration is stored and analysed. Finally, the performance comparison is carried out.

\subsection{Meta-Model Validation}

In this step, the Meta-Model validation is performed, using the Meta-Data as training data and a new set of test images. The test set is made up of $30 \%$ of the images of each dataset, selected randomly, the label that identifies which is the best classification and characterization method for each type of image is the same one recognized for the dataset in the step of the Classification Method Selection (Section 4.1), however, this information is used only to verify the result of the classification given by the MetaModel.

The step begins with the creation of the test set, containing the images and the class labelling information. Then the performance of Meta-Model is stored and analysed.

\section{RESULTS}

In this section, the performance of different configurations for CNN is evaluated and compared for the classification task, and the performance of the Meta-Model with a set of test images is also evaluated. The processing of the data and the applications of the CNN were carried out using Waikato Environment for Knowledge Analysis (WEKA) (Witten, 2016).

For the construction of the Meta-Data, $70 \%$ of the images of each dataset was randomly selected: 1260 from NEU surface dataset, 756 from shear pad of wagon train dataset, 62 from welds X-ray dataset, 50 from Aluminum wheel Xray dataset, finally 210 from Human Face dataset. In total the training set that is part of the Meta-Data is formed by 2338 images, the other component of the Meta-Data are the step of classification and characterization methods identified in the step of Classification Method Selection (Section 4.1) for each dataset.

Table 1 shows the results of the Classifier Evaluation for Meta-Model step. Accuracy, area under the ROC curve
(AUC) (Bradley, 1997) and F-measure (Goutte, 2005) are used as measures of the performance of each configuration of CNN classification method.

Table 1. Test result for different CNN configurations.

\begin{tabular}{cccc} 
Method & Accuracy $\%$ & AUC \% & F-measure \% \\
\hline Lenet & $\mathbf{9 9 . 3}$ & $\mathbf{9 9 . 9}$ & $\mathbf{9 9 . 3}$ \\
VGG16 & 54.3 & 0.5 & 0.7 \\
Proposal 1 & 96.7 & 99.8 & 96.7 \\
Proposal 2 & 97.7 & $\mathbf{9 9 . 9}$ & 97.6 \\
Proposal 3 & 98.2 & 99.7 & 98.2 \\
Proposal 4 & 95.8 & $\mathbf{9 9 . 9}$ & 95.5 \\
\hline
\end{tabular}

When analyzing the results of Table 1 it is possible to find: First, the VGG16 configuration presents a loss of the relevant features, which starts from the fourth layer and becomes critical in the ninth layer. Second, when comparing proposals $1,2,3$ and 4 , it is found that there is a loss of relevant information when using a larger convolution matrix, conversely it is possible to increase the size of the subsampling matrix without losing relevant information, however, when using both resources, as in the case of proposal 4 , it is found that the loss of information increases, indicating that the loss of information due to the change in the size of the subsampling matrix increases. Finally, the Lenet configuration is the most suitable to be used as a classifier of the Meta-Model, given its superior value of $\mathrm{F}$-measure, which indicates a better classification given the unbalanced nature of the classes.

For Meta-Model Validation step, a test set is constructed consisting of 1004 images taken randomly from the datasets: 540 from NEU surface dataset, 325 from shear pad of wagon train dataset, 26 from welds X-ray dataset, 22 from Aluminum wheel X-ray dataset and 90 from $\mathrm{Hu}-$ man Face dataset. 96\% accuracy, 99.7\% AUC and 96.5\% F- measure was obtained, in practice the results show that the use of Meta-Learning is efficient to identify the most appropriate methods of image classification to perform tasks such as quality control in products and welding, identify defective parts or expressions on the faces of the staff.

\section{CONCLUSION}

In this work a new methodology based on Meta-learning has been presented for the identification of the most suitable methods of classification of industrial images, for this purpose five dataset and six different configurations of parameters for CNN were used. Based on the results it is possible to conclude that the proposed methodology is effective for the identification of the most appropriate methods of classification and characterization in industrial images. The CNN method is effective as a characterization and classification method for the Meta-model. The use of Meta-Learning allows to change or update the information of classification and characterization methods quickly and easily, since the characterization is done only on the images under study. When comparing the different configurations for CNN, it is concluded that for the classification of the images under study it is necessary to use two convolution layers.

For future work, it is planned to expand Meta-Data by including new types of images and make a comparative 
analysis of performance between CNN and different classification techniques based on Machine Learning that use characterization methods.

\section{REFERENCES}

Amiri, S. M. et al. (2014) 'Human action recognition using meta learning for RGB and depth information', in 2014 International Conference on Computing, Networking and Communications, ICNC 2014.

Baldassari Soares, L. et al. (2017) 'Sistema de Visão Computacional para Análise Geométrica de Cordões de Solda', in XIII Simpósio Brasileiro de Automação Inteligente, 2017.

Ben Driss, S. et al. (2017) 'A comparison study between MLP and convolutional neural network models for character recognition', SPIE, 2017.

Bradley, A. P. (1997) 'The use of the area under the ROC curve in the evaluation of machine learning algorithms', Pattern Recognition, 1997.

Brazdil, P. and Giraud-Carrier, C. (2018) 'Metalearning and Algorithm Selection: progress, state of the art and introduction to the 2018 Special Issue', Machine Learning. 107(1), pp. 1-14. Springer US, 2018.

Campos, G. F. C., Barbon, S. and Mantovani, R. G. (2017) 'A meta-learning approach for recommendation of image segmentation algorithms', Proceedings - 2016 29th SIBGRAPI Conference on Graphics, Patterns and Images, pp. 370-377, SIBGRAPI 2016, 2016.

Chaib, S., Yao, H., Gu, Y., and Amrani, M. (2017). 'Deep feature extraction and combination for remote sensing image classification based on pre-trained CNN models', Proceedings of SPIE - The International Society for Optical Engineering,July 2017.

Cheplygina, V. et al. (2017) 'Exploring the Similarity of Medical Imaging Classification Problems', in Lecture Notes in Computer Science (including subseries Lecture Notes in Artificial Intelligence and Lecture Notes in Bioinformatics). 2017.

Cunha, T., Soares, C. and de Carvalho, A. C. P. L. F. (2018) 'Metalearning and Recommender Systems: A literature review and empirical study on the algorithm selection problem for Collaborative Filtering', Information Sciences. 423, pp. 128-144. Elsevier Inc., 2018.

Ekman, P. and Friesen, W. V. (1976) 'Measuring facial movement', Environmental Psychology and Nonverbal Behavior, 1976.

Deng, Z. et al. (2016) 'Efficient kNN classification algorithm for big data', Neurocomputing,2016.

Goutte, C. and Gaussier, E. (2005) 'A Probabilistic Interpretation of Precision, Recall and F-Score, with Implication for Evaluation BT - Lecture Notes in Computer Science', in Lecture Notes in Computer Science, 2005.

Huiskes, M. J. and Lew, M. S. (2008) 'The MIR flickr retrieval evaluation', ACM International Conference on Multimedia Information Retrieval (MIR'08), Vancouver, Canada, 2008.

Huiskes, M., Thomee, B. and Lew, M. (2010) 'New trends and ideas in visual concept detection: the MIR flickr retrieval evaluation initiative', Proceedings of the international conference on Multimedia information retrieval, pp. 527-536, 2010.

Jolliffe, I. T. (2002) Principal Component Analysis, Second Edition, Encyclopedia of Statistics in Behavioral
Science.

Lecun, Y., Bengio, Y. and Hinton, G. (2015) 'Deep learning', Nature Methods,2015.

Mantovani, R. G. et al. (2015) 'Meta-learning recommendation of default hyper-parameter values for SVMs in classifications tasks', in CEUR Workshop Proceedings, 2015.

Mery, D. and Filbert, D. (2002) 'Automated flaw detection in aluminum castings based on the tracking of potential defects in a radioscopic image sequence', IEEE Transactions on Robotics and Automation.

Mery, D. (2011) 'Automated detection in complex objects using a tracking algorithm in multiple X-ray views', in IEEE Computer Society Conference on Computer Vision and Pattern Recognition Workshops, 2011.

Mery, D.; Riffo, V.; Zscherpel, U.; Mondragón, G.; Lillo, I.; Zuccar, I.; Lobel, H.; Carrasco, M. (2015): GDXray: The database of X-ray images for nondestructive testing. Journal of Nondestructive Evaluation, 34.4:1-12, 2015.

Pimentel, B. A. and de Carvalho, A. C. P. L. F. (2019) 'A new data characterization for selecting clustering algorithms using meta-learning', Information Sciences., 477, pp. 203-219, Elsevier Inc.,2019.

Rabiner, L. R. (1989) 'A Tutorial on Hidden Markov Models and Selected Applications in Speech Recognition', Proceedings of the IEEE, 1989.

Rahman, M. M. and Bhattacharya, P. (2017) 'Biomedical image classification with multi response linear regression (MLR) as meta-learner combiner and its effectiveness on small to large data sets', in Proceedings - 2016 International Conference on Computational Science and Computational Intelligence, CSCI 2016.

Reynolds, D. (2015) 'Gaussian Mixture Models', in Li, S. Z. and Jain, A. K. (eds) Encyclopedia of Biometrics. pp. 827-832. Springer US, Boston, 2015.

Rivera, H. et al. (2017) 'Development of an Automatic Expression Recognition System Based on', XIII Simpósio Brasileiro de Automação Inteligente, pp. 615-620, 2017.

Rocha, R. et al. (2017) 'Avaliação de técnicas de Deep Learning aplicadas à identificação de peças defeituosas em vagões de trem', Workshop of Industry Applications (WIA) in the 30th Conference on Graphics, Patterns and Images (SIBGRAPI'17). in Clua, E. and Pádua, F. L. C. (eds), Niterói, RJ, Brazil, 2017.

Simonyan, K. and Zisserman, A. (2014) 'Very deep ConvNets for large-scale image recognition', CoRR, 2014.

Smith, M. R. et al. (2014) 'Recommending learning algorithms and their associated hyperparameters', in CEUR Workshop Proceedings, 2014.

Song, K. and Yan, Y. (2013) 'A noise robust method based on completed local binary patterns for hot-rolled steel strip surface defects', Applied Surface Science, vol. 285, pp. 858-864, 2013.

Vapnik,V.N.(1995)'The Nature of Statistical Learning Theory', Springer, 1995.

Weiss, K., Khoshgoftaar, T. M. and Wang, D. D. (2016) 'A survey of transfer learning', Journal of Big Data, 2016.

Witten, I. H. et al. (2016) 'Data Mining: Practical Machine Learning Tools and Techniques', pp. 1-621, Elsevier Inc, 2016. 